Gilbert F. White

David J. Bradley

Anne U. White

Illustrated by Tag Ahmed

\title{
Drawers of Water
}

\section{Domestic WaterUse in East Africa}

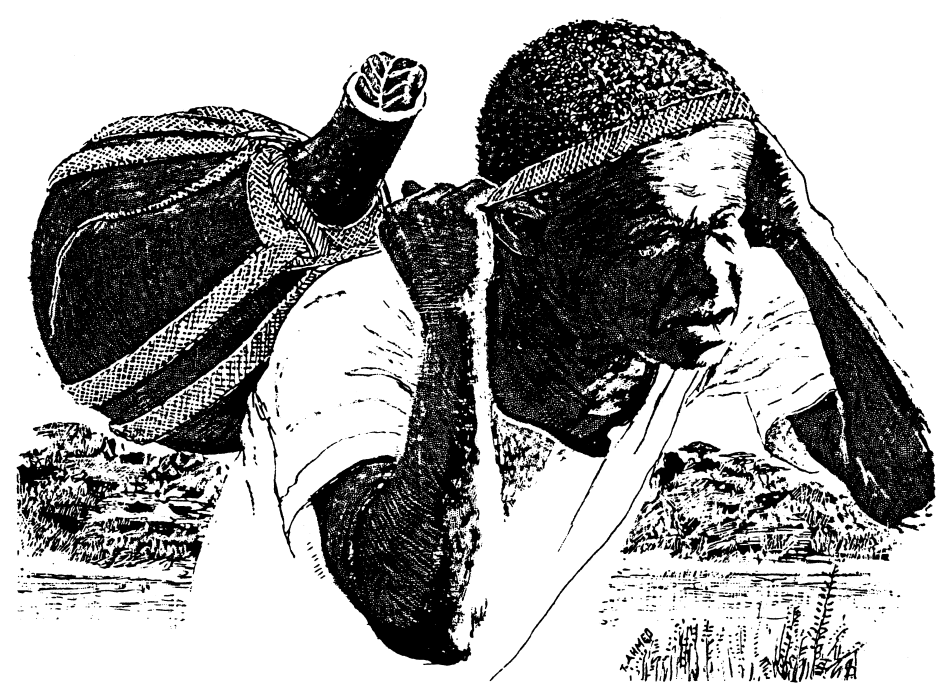

\section{Total Social Costs}

$\ldots$

\section{THE INTERRELATION OF HEALTH AND IMPROVEMENTS}

The epidemiological basis for the prevention of infections related to water by improving supplies may be summarized in the following way. The general model is illustrated in figure 7.1. Under conditions of appalling water supply the amount of illness is $A$. Increasing the volume supplied will decrease the morbidity until a point is reached where no matter how much more water is supplied the situation will remain static with $V$ units of disease. On the other hand, improving water quality alone will reduce the disease amount to level $P$. Improving all aspects of water supply will, at optimal purity and volume, reduce the disease level to $M$. This residual amount will be unaffected by changes in water supply. The values of $A, M, P$, and $V$ will depend on the physical and social environment and on the disease being considered. An aggregate model for all disease in a particular area 
THE GENERAL CASE
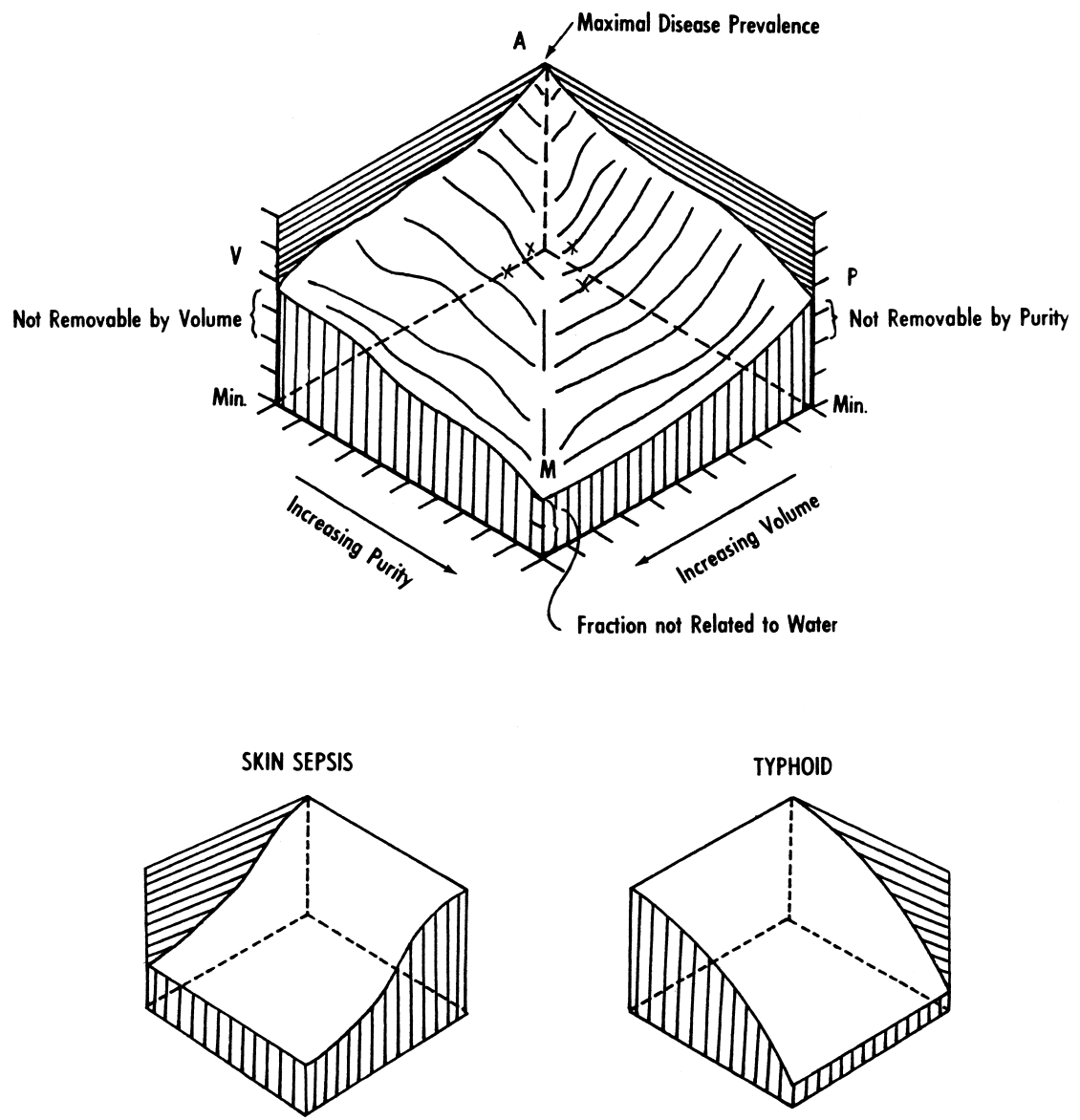

Fig. 7.1. Diagrams of relation of disease prevalence to water: the general case; skin sepsis; and typhoid.

will have a particular form depending on the environment. A similar model for improvement costs may be constructed (fig. 7.2), and the two may be compared. Quantitative data for such disease models are unavailable, but qualitatively the position is as follows.

The waterborne diseases in the strict sense are due to polluted supplies. The classical waterborne diseases have a low infecting dose of organisms and may produce dramatic epidemics following relatively light pollution of a large common source. They reach their greatest importance in urban areas where the number of households 
per source is highest and for their prevention require completely pure supplies. The nonclassical waterborne infections are those requiring a larger infecting dose of microbes and are usually commoner diseases than the preceding ones, though often not waterborne in more developed countries. They are transmitted under conditions of heavy pollution even when sources do not supply large groups of households, and they may produce sporadic disease. We know little about their extent but suspect that they are most important in urban areas with very heavy pollution of unimproved sources. They are prevented by moderate protection of sources short of absolute sterility.

The water-washed diseases are of two types. The bulk of such diseases as seen in the outpatient clinic are superficial infections of the skin and eyes. These reach their peak in dry areas with both a scarcity of water for washing and a dusty environment, though a generally

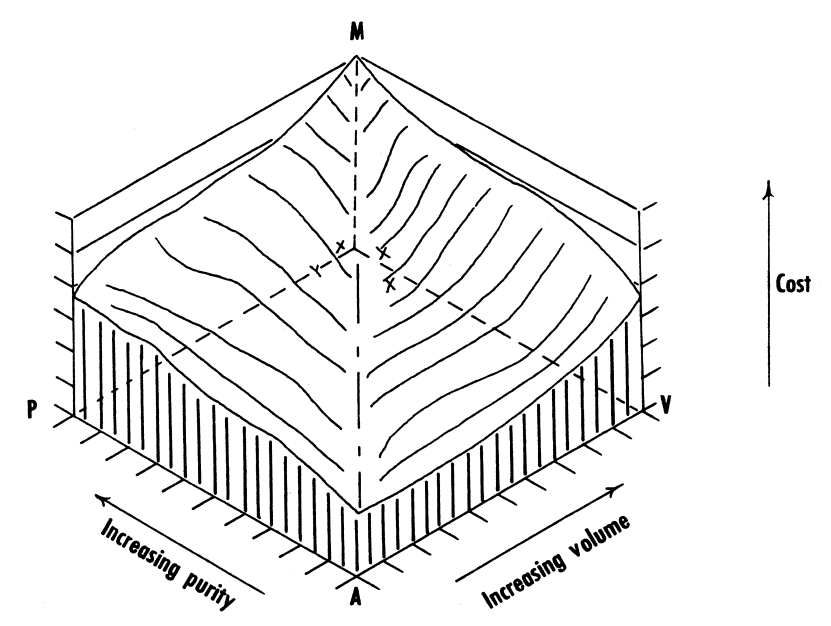

Fig. 7.2. Diagram of relation of cost of provision to water purity and volume

unsanitary habitat makes matters worse. These are clearly of the water-washed category and the prime need in most rural areas is for a more accessible supply of greater volume. Purity is a secondary consideration and should not delay attempts to increase the quantities available.

The diarrheal diseases also seem to diminish when water supplies are made more accessible. Their precise etiology is still far from clear and other factors are important, so that there are variations in prevalence between areas with comparable water supplies. A hot, dry climate and an unsanitary environment are both associated with much 
diarrhea, which is therefore common both in crowded urban and arid rural areas.

Where the population density rises, whether in towns or in some densely inhabited rural areas, purity becomes of greater relative importance, not only because more people use each common source but also because dense rural settlement tends to be in high-rainfall areas. On the basis of evidence presented elsewhere, gastroenteric disorders appear to have both water-washed and waterborne components and reach their peak in overcrowded periurban zones where, fortunately, most can be done about them, though so far municipal authorities have paid more attention to purity than to availability of improved supplies. The diarrheal diseases and typhoid cause hospital admissions and death far more often than the superficial water-washed infections; they affect treatment costs more and also provide the bulk of lost work time and the economic losses from premature death.

The water-multiplied and other water-based infections are most prevalent where contact with the sources is intense and localized. This is the case in hot dry areas with few and rather small sources. They can be controlled by rather specialized measures to reduce contact with the sources or by general procedures leading to the same effect.

The infections carried by water-dependent insects again are of two types. The vectors which breed in water are unlikely to be greatly affected either way by changing domestic water sources, but the waterside biting vectors are often closely associated with water collecting. They are mainly a rural problem; some are important chiefly in arid areas but others are associated with more rapid streams from high ground. Both groups are most affected by provision of new sources or by specialized preventive measures.

Particular water-multiplied infections and diseases with waterrelated insect vectors are local problems and can best be overcome by particular local solutions, but these may be organized under national programs.

The position regarding infective disease and water supplies is summarized in table 7.1. Here the diseases are grouped in the four classes according to their relation to water. Each disease is rated by commonness, severity, and chronicity. The degree of effect of water volume and of water purity is indicated, and the infection route (drinking or bathing) is noted. Finally, an estimate is made of the percentage of reduction in the morbidity which might be expected from thorough improvement of the water supply. This is largely guesswork based on a wide experience of East African conditions.

If we aggregate total health cost for a typical tropical country, the 


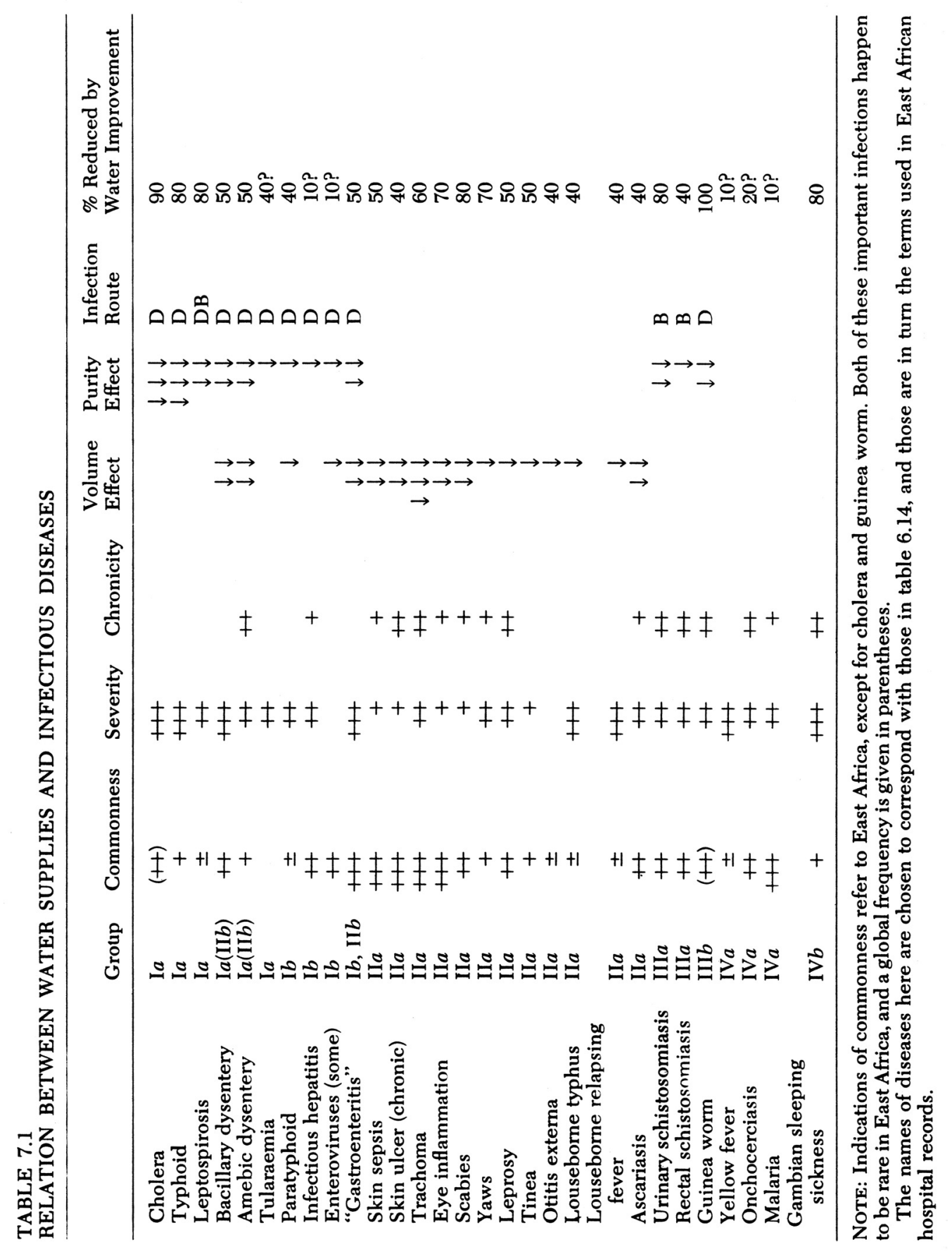


relation between water and health is as shown in figure 7.1. A similar diagram of the cost of improvements and transport (viewed from the opposite corner of the diagram) is figure 7.2. Maximum cost is reached at level $M$. The nation that is wary of overprecise economic arguments and has limited resources will aim for supplies somewhere in the zones marked $X$, where value for money is greatest. In our view it may perhaps correspond to fairly pure single-tap, or single-tap with shower, piped water to the home, but this subject is in great need of field study.

\section{Seven Model Habitats}

Clearly, some improvements are so costly that they are not feasible in certain environments, and the health benefits from a given improvement will also vary with the environment-a bath that might prevent skin infection in the hot desert could probably give an Eskimo pneumonia, though the Eskimo, too, suffers from inadequate supplies in many ways. Therefore seven model East African habitats are considered in detail. Since real environments form a continuum, we have specified the characteristics of each model "site" as indicated in table 7.2. Two are urban, three are rural with dispersed settlement, and two more are rural nucleated settlements. The last two are scarcely found in much of East Africa at present, but if the plans of the Tanzania and Uganda governments reach fruition they will increasingly become a feature of East African life, and, indeed, it is difficult to see how many of the developmental aims of these nations can be realized without some degree of nucleation of settlements.

\section{Health Cost Estimates}

The estimates of health cost in different environments, and the effect of various types of improvement in lowering them, are of course very arbitrary guesses, and one may ask why they are made. By its action in deciding on a particular type of water improvement, a government is implying a certain level of health cost, and it therefore seemed desirable to attempt these estimates from the disease pattern. Health costs are measured on an arbitrary centile scale on which 100 units represents the amount of health cost due to water-related disease under limiting conditions of water availability in a semiarid region. Zero units of health cost represents the amount of disease under conditions of ample free pure water by a multiple outlet system in the particular environment being considered: circumstances under which changes in water supply can effect no further improvements in health. Thus, in an environment where the health cost was initially 


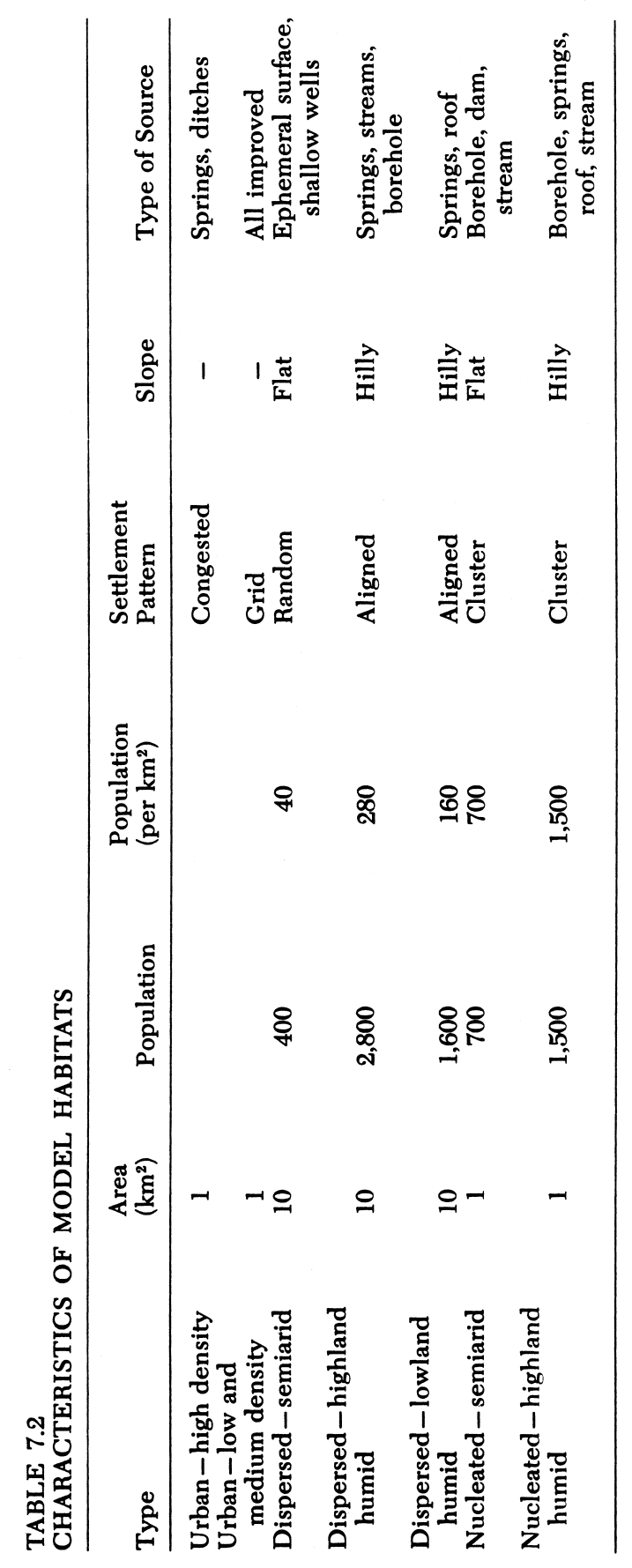


80 units in the absence of water improvements, a reduction to 40 units would indicate that the relevant improvement had taken health costs halfway to those achieved under ideal circumstances of water supply. Health cost is conceived of as aggregating the costs of morbidity, mortality, currently available treatment, and economic loss along the lines discussed in chapter 6 .

\section{Urban High Density}

The dense urban settlement may have a disease level of 95: diarrheal diseases worse than in dispersed settlement but diseases spread by water-related vectors less abundant. Figure 7.3 illustrates the range of aggregate social costs. Class 1 improvements can only be roof supplies, and these, not adequate for the entire year but giving an increased and unpolluted supply, might reduce disease to 40 units. In class 2 spring improvement can effect little in the face of such fecal pollution and a fall to 75 units is optimistic, but boreholes could give 55 units if unpolluted. Differences in the length of bars for classes 1 and 2 correspond to different types of improvement, for example, boreholes versus springs. Piping spring water is not feasible; nor is borehole water likely to be piped in this environment-if it was, a fall to 50 units might occur; it would depend on where the pipes reached. Standpipes from a pure municipal supply (class 4) might reduce the disease to 45 units, and a further considerable fall to 15 units could perhaps be obtained in class 5 provided a shower mechanism was included. Class 6 , as at all sites, would by definition reduce the disease to zero.

\section{Low and Medium Density Urban}

Low and medium density urban areas already have class 6 installations in East Africa. It is unrealistic to suggest other classes of improvement. However, a revised pricing structure that greatly reduced uses for car washing and lawn watering would not detectably raise the disease rate.

\section{Dispersed Semiarid}

The dispersed semiarid area would have a high disease rate (100). All four groups of water-related disease would occur. Individual improvements would scarcely be practical-roof water would be inadequate for the year and, because of the intermittent rainfall, would require a vast storage capacity. At best therefore it might halve the disease rate. A smaller reduction could be expected from a borehole, provided it was used and was not farther from the homes than the initial source. 
A dam would be no more effective on its own, but a pipeline would reduce the disease further. Further stages of improvement (4-6), though effective in improving health, would be prohibitively expensive to install.

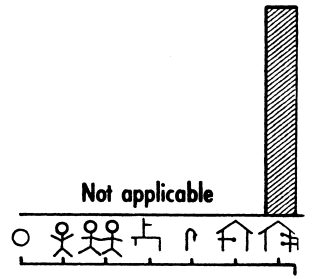

LOW AND MIDDLE DENSITY URBAN

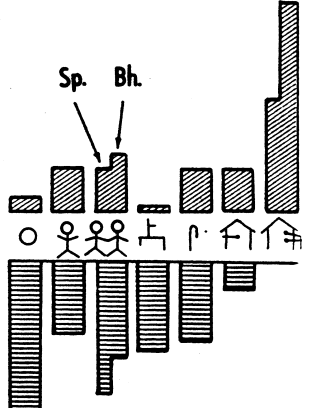

HIGH DENSITY URBAN
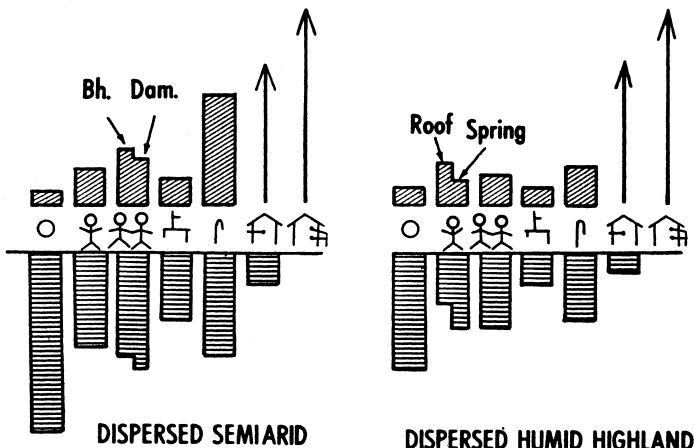

DISPERSED HUMID HIGHLAND

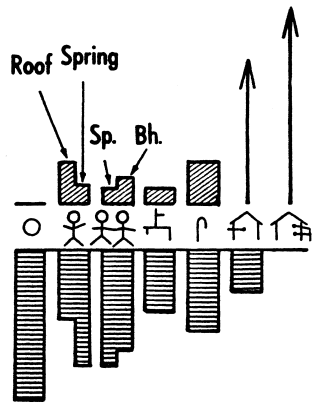

DISPERSED LOWLAND

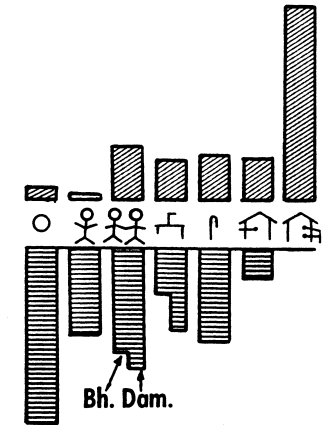

NUCLEATED SEMIARID

NUCLEATED HUMID

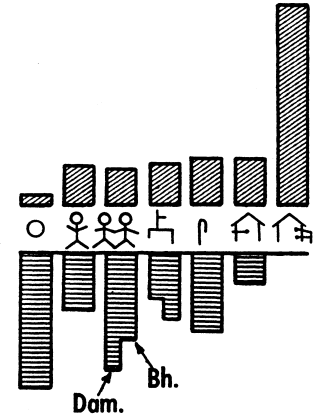

冒 Different Improvements

Fig. 7.3. Measures of health and use costs for water improvements in seven model habitats. 


\section{Dispersed Highland Humid}

An area of the dispersed highland humid type might have an initial disease level of perhaps 60 units, due more to source pollution than to water shortage. Roof water would effect considerable improvement, and individual source protection some. Community action in preventing pollution would also be effective. Piping water to houses from a relatively protected source would achieve many of the desired gains; even if financially feasible, pure water from standpipes would be a retrograde step from the preceding stage, though completely pure water piped to the houses is ideal.

\section{Dispersed Lowland Humid}

In the dispersed lowland humid environment individual action in collecting roof water or protecting a nearby source for use of one or two households yields health improvements from perhaps 80 units to 35 (roofs) or 60 units. Community action gives a similar result from spring protection, perhaps greater improvement from borehole water. A distribution system on this gently undulating landscape requires an initial pumping system even if delivery from the storage tank is by gravity. The increased availability of water from pipes reduces health costs. As in the highlands, chlorinated systems of classes 4-6 are scarcely practical propositions, though all but the standpipes would be desirable from a health viewpoint.

\section{Nucleated Semiarid}

The nucleated semiarid village from a health viewpoint would resemble the dispersed settlement in a similar climate except that effects of source pollution would be increased and distance to sources would be less. The main effect of nucleation is on the cost of improvements of classes 4-6. These are practicable in a nucleated village, but almost inconceivable otherwise.

\section{Nucleated Humid}

Results very similar to those in nucleated semiarid habitats can be expected from improvements to the nucleated village in a humid area. The only notable difference would be that in class 1 roof supplies would give perennial pure water.

It is necessary to repeat the warning not to overinterpret the crude guesses of health cost represented in figure 7.3. The important points to notice are that often a modest change in improvement costs may considerably affect health, whereas in other cases very expensive water improvements may produce few health benefits. Class 3 benefits could often be further increased by extending the piping from the yard to the house itself and adding a shower connection. 
A summary of these relationships is presented in table 7.3. Concrete figures are not given, but relative importance is indicated by symbols. Separate estimates are omitted for the two habitats having nucleated rural settlement: the conditions of disease so nearly approximate those in dispersed settlement with similar climate and terrain that there is little point in repeating them.

\section{TABLE 7.3}

GENERALIZED CHARACTERISTICS AND NEEDS OF DIFFERENT HABITATS IN RELATION TO DOMESTIC WATER SUPPLIES, IN EAST AFRICA

\begin{tabular}{|c|c|c|c|c|c|}
\hline & \multicolumn{2}{|c|}{ Urban } & \multicolumn{3}{|c|}{ Rural } \\
\hline & $\begin{array}{l}\text { Low } \\
\text { and Medium } \\
\text { Density }\end{array}$ & $\begin{array}{l}\text { High } \\
\text { Density }\end{array}$ & $\begin{array}{l}\text { Semi- } \\
\text { arid }\end{array}$ & $\begin{array}{l}\text { High- } \\
\text { land } \\
\text { Humid }\end{array}$ & $\begin{array}{l}\text { Low- } \\
\text { land } \\
\text { Humid }\end{array}$ \\
\hline \multicolumn{6}{|l|}{ Characteristics } \\
\hline High temperature & + & + & + & - & + \\
\hline Humidity & + & + & - & + & + \\
\hline Altitude & & & - & + & - \\
\hline Slope & - & - & - & + & - \\
\hline People per unit area & + & $+H$ & + & + & + \\
\hline People per source & ++ & $+1+$ & ++ & + & + \\
\hline Source water/total water & + & + & $+1+$ & - & - \\
\hline Sources per unit area & - & + & - & + & + \\
\hline Financial resources available & $+1+$ & + & - & + & - \\
\hline Distance to water point & - & + & $+1+$ & + & + \\
\hline Source pollution & - & $+H$ & + & + & + \\
\hline \multicolumn{6}{|l|}{ Dangers of disease } \\
\hline Classical waterborne & + & $+1+$ & + & + & + \\
\hline Nonclassical waterborne & - & + & + & + & + \\
\hline Water-washed skin & - & + & $+1+$ & + & + \\
\hline Water-washed diarrheal & - & $+H$ & $+1+$ & + & + \\
\hline Water-based percutaneous & - & + & $+1+$ & + & + \\
\hline Water-based oral & - & - & $+1+$ & - & - \\
\hline Water-breeding & + & + & + & ++ & + \\
\hline Water-biting & - & - & $+1+$ & + & + \\
\hline \multicolumn{6}{|l|}{ Needs } \\
\hline Present fulfillment & $+H$ & - & - & - & - \\
\hline Moderate purity & & $+1+$ & + & + & ++ \\
\hline Great purity & & $+1+$ & & & \\
\hline Volume & & + & $+H$ & & \\
\hline Increased accessibility & & + & $+H+$ & + & ++ \\
\hline New sources & & & $+1+$ & & \\
\hline Suppression of alternative & & & & & \\
\hline sources & & + & & & \\
\hline Special measures & & & ++ & + & \\
\hline Priorities & & 1 & 1 & 2 & 3 \\
\hline \multicolumn{6}{|l|}{ Costs } \\
\hline Moderate improvement & & + & $+1+$ & + & + \\
\hline Maximum improvements & & + & $+1+$ & + & ++ \\
\hline
\end{tabular}

